Supporting Information

\title{
Understanding Covalent versus Spin-Orbit Coupling Contributions to Temperature Dependent Electron Spin Relaxation in Cupric and Vanadyl Phthalocyanines
}

\author{
Alec H. Follmer†, Ryan D. Ribson†, Paul H. Oyala, Grace Y. Chen, Ryan G. Hadt* \\ Division of Chemistry and Chemical Engineering, Arthur Amos Noyes Laboratory of \\ Chemical Physics, California Institute of Technology, Pasadena, California 91125, United \\ States \\ †Co-first author \\ *Corresponding Authors: rghadt@caltech.edu
}




\section{Table of Contents}

Figure S1. Powder XRD of VOPc:TiOPc and CuPc:ZnPc...............................................S3

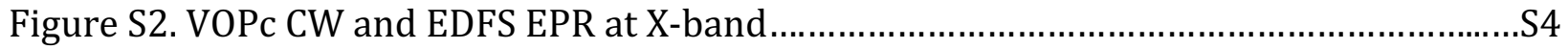

Figure S3. VOPc Temperature dependence of EDFS $T_{1}$ and $T_{m}$ EPR X-band............................S5

Figure S4. VOPc Temperature dependence of EDFS T1 and Tm EPR Q-band .......................S6

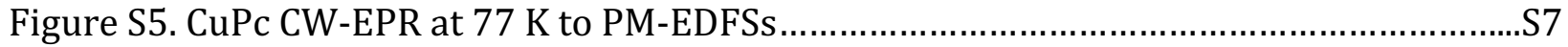

Figure S6. CuPc Temperature dependence of EDFS T1 and Tm EPR Q-band.......................S8

Figure S7. Inversion recovery and Echo Decay Spectra with fits....................................S9

Figure S8. 3D comparison of CuPc 1:1000 $T_{1}$ and $T_{m}$ relaxation times...............................

Figure S9. 3D comparison of VOPc 1:1000 $T_{1}$ and $T_{m}$ relaxation times...............................S11

Figure S10. Comparison of the stretching term, in CuPc and VOPc for $T_{1}$ and $T_{m} \ldots \ldots \ldots \ldots \ldots . . . . . . . .19$

Figure S11. CuPc:ZnPc (IP) temperature dependence of EDFS, $T_{1}$, and $T_{m}$ EPR X/Q-band....S20

Figure S12. X-band CW-EPR at $77 \mathrm{~K}$ of the CuPc:ZnPc of the independent preparation......S22

Table S1. Best-fit parameters of the simulated X-band EPR of VOPc:TiOPc ..........................S4

Table S2. Best-fit parameters of the simulated X-band EPR of CuPc:ZnPc...........................S7

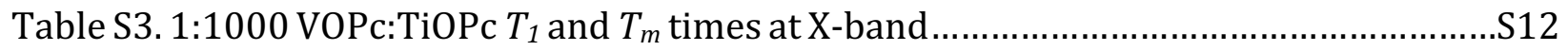

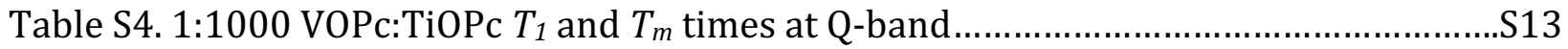

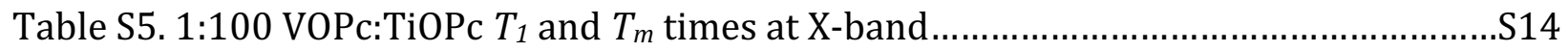

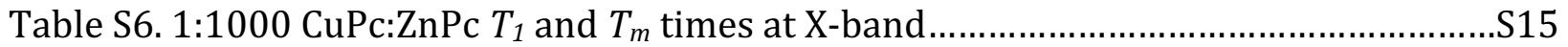

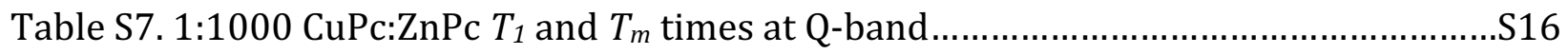

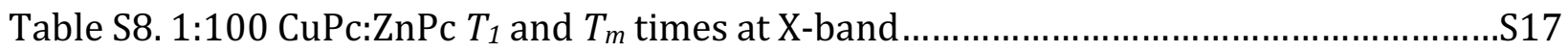

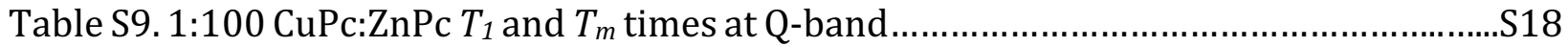



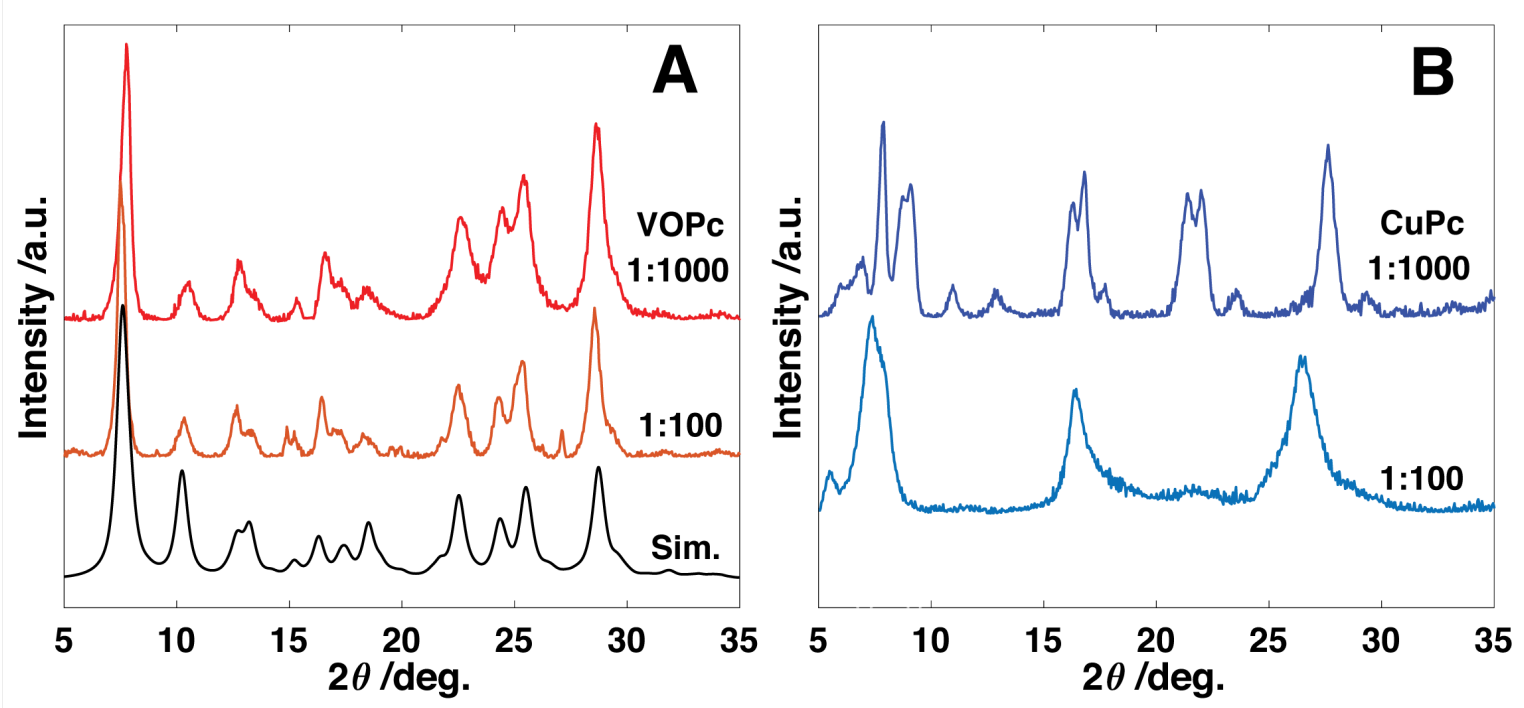

Figure S1. (A) Comparison between PXRD patterns of VOPc:TiOPC at concentrations of 1:1000 (top) 1:100 (middle) and simulated Type II VOPc (bottom). (B) Comparison between the PXRD patterns of CuPc:ZnPc 1:1000 (top) and 1:100 (middle) and simulated $\beta$-CuPc (bottom).

The PXRD patterns of both the 1:1000 and 1:100 VOPc:TiOPc mixtures shown in Figure S1 are consistent with previously reported diffraction data for the type-II polymorph and are well reproduced by simulation. This indicates structural phase homogeneity of the vanadyl samples. The PXRD patterns of the 1:1000 and 1:100 CuPc:ZnPc dispersions show distinct patterns between samples and do not strictly match either the reported $\alpha$ - or $\beta$-CuPc diffraction patterns. ${ }^{1}$ 

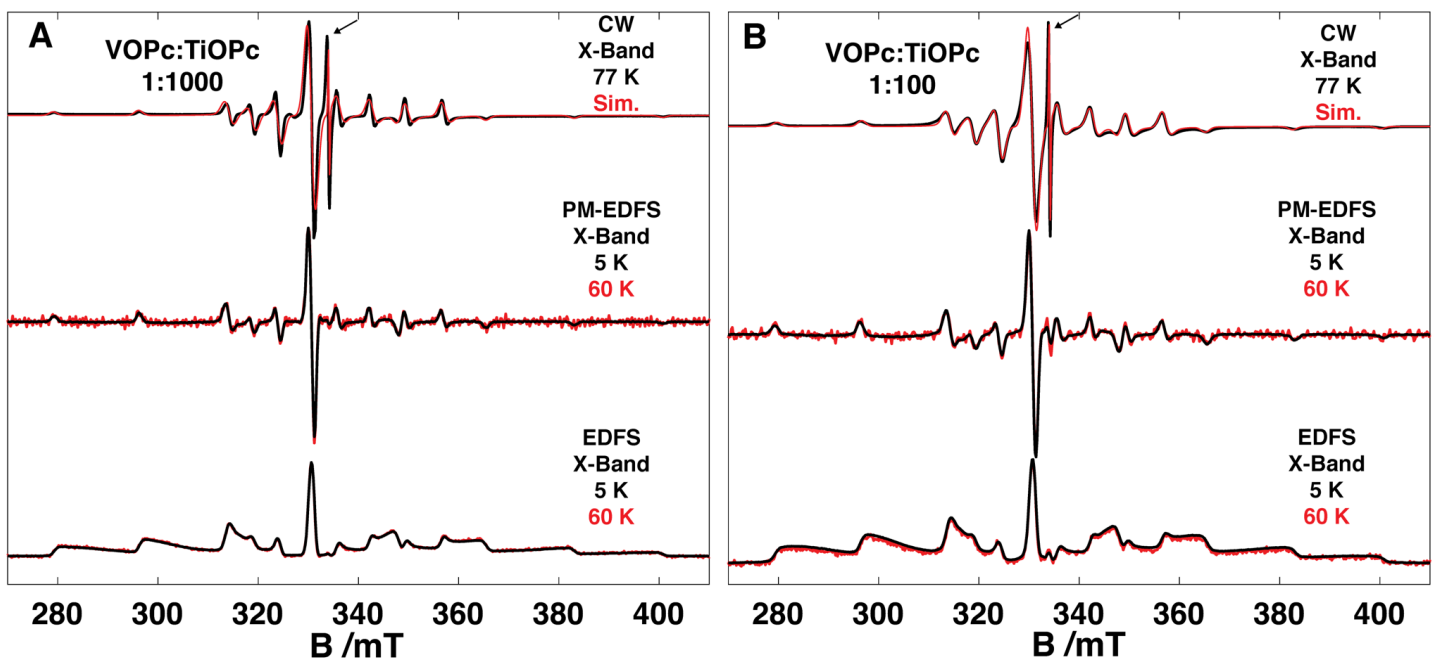

Figure S2. Comparison between CW X-band EPR at $77 \mathrm{~K}$ to pseudo-modulated echo-detected field sweeps (PM-EDFSs) and the associated field sweeps at $5 \mathrm{~K}$ (black) and $60 \mathrm{~K}$ (red) of (A) VOPc 1:1000 and (B) 1:100. The additional radical species is indicated by an arrow.

\begin{tabular}{|l|l|l|}
\hline & $\mathbf{1 : 1 0 0 0}$ VOPc:TiOPc & $\mathbf{1 : 1 0 0 ~ V O P c : T i O P c ~}$ \\
\hline $\mathbf{g}_{\|}$ & 1.968 & 1.968 \\
\hline $\mathbf{g}_{\perp}$ & 1.988 & 1.988 \\
\hline $\mathbf{A}_{\|}(\mathbf{M H z})$ & 478.6 & 473.6 \\
\hline $\mathbf{A}_{\|}\left(\mathbf{c m}^{-1}\right)$ & $160 \times 10^{-4}$ & $158 \times 10^{-4}$ \\
\hline $\mathbf{A}_{\perp}(\mathbf{M H z})$ & 167.6 & 167.9 \\
\hline $\mathbf{A}_{\perp}\left(\mathbf{c m}^{-1}\right)$ & $56 \times 10^{-4}$ & $56 \times 10^{-4}$ \\
\hline
\end{tabular}

Table S1. Best-fit parameters of the simulated EPR spectra in Figure S2 used to reproduce the EPR spectra $\mathrm{T}=77 \mathrm{~K}$.

The CW-EPR of the 1:1000 and 1:100 samples of VOPc are similar. The 1:100 sample exhibits slightly broader features, presumably due to increased dipolar interactions with other vanadyl species at this higher concentration. The EDFSs of the two dilutions at both $5 \mathrm{~K}$ and $60 \mathrm{~K}$ are similar and consistent with literature reports. The PM-EDFS spectra lack the sharp radical feature present in the CW EPR (although it may slightly appear in the 1:100 spectrum), indicating that the spins responsible for this signal relax significantly slower than the shot repetition time of the spin echo detection experiment. This feature has previously been attributed to organic radical impurities that are consistently found even in diamagnetic phthalocyanine matrices. ${ }^{2,3}$ In both 1:1000 and 1:100 samples, the radical feature was fit to a $\mathrm{g}=2.0$ and $\sim 1.0 \%$ weight in the CW spectra. 

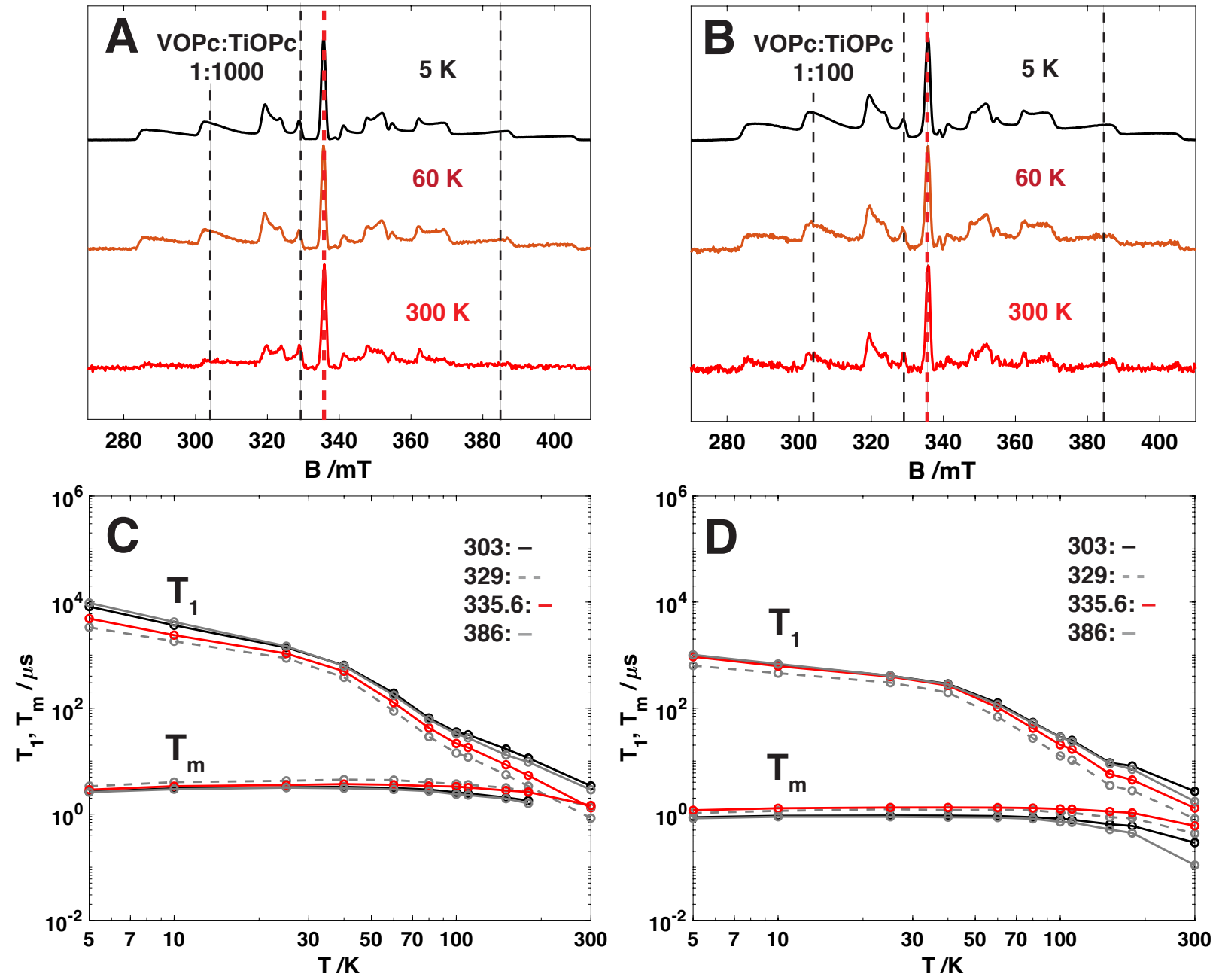

Figure S3. Echo-detected EPR X-band field sweeps of (A) VOPc:TiOPc 1:1000 and (B) 1:100 at $5 \mathrm{~K}, 60 \mathrm{~K}$, and $300 \mathrm{~K}$. Dashed lines indicate field positions where relaxation data were collected. Comparison between the field position dependent behavior of the $T_{1}$ and $T_{m}$ relaxation times from 5 to $300 \mathrm{~K}$ of VOPc:TiOPc (C) 1:1000 and (D) 1:100. 

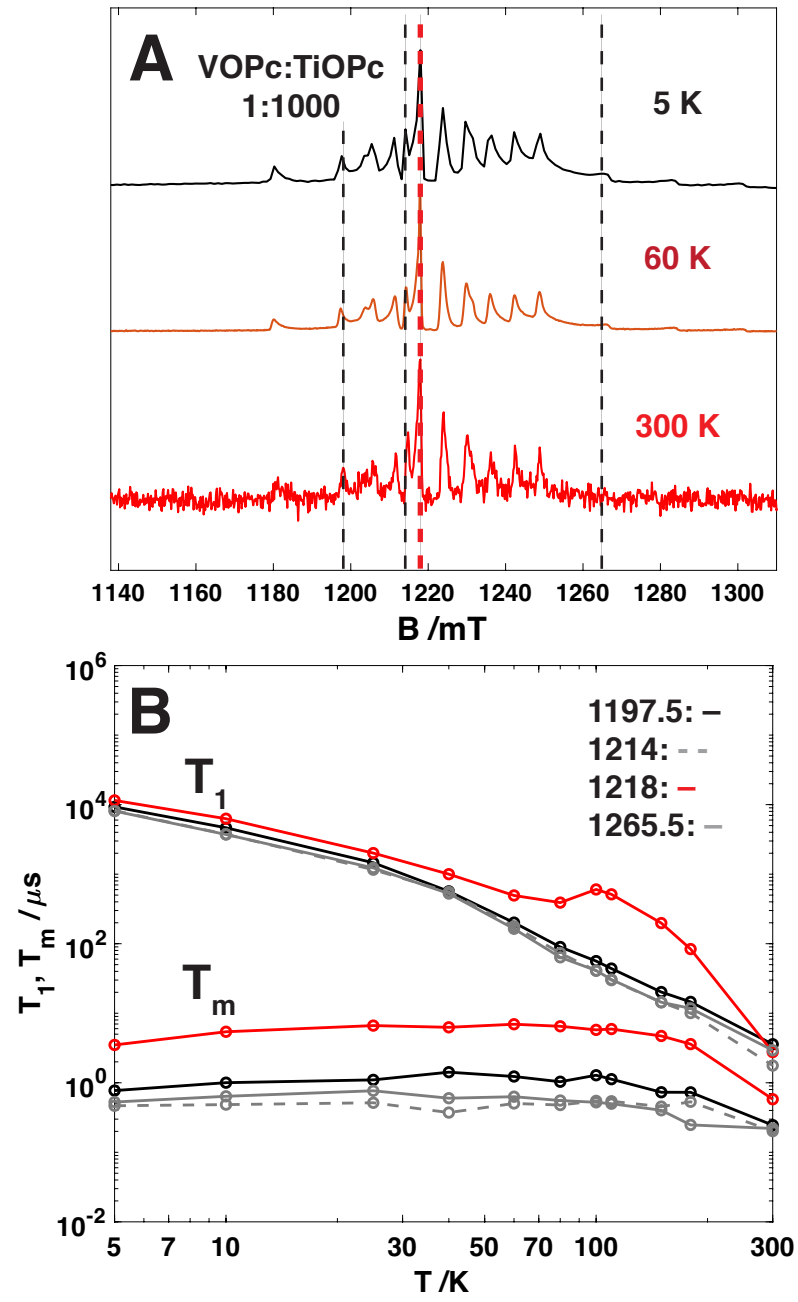

Figure S4. EDFS EPR Q-band of (A) VOPc 1:1000 at 5 K, 10 K, and 60 K. Dashed lines indicate field positions where relaxation data was collected. Comparison of the field position dependent behavior of the $T_{1}$ and $T_{m}$ relaxation times from 5 to $300 \mathrm{~K}$ of VOPc (B) 1:1000. 

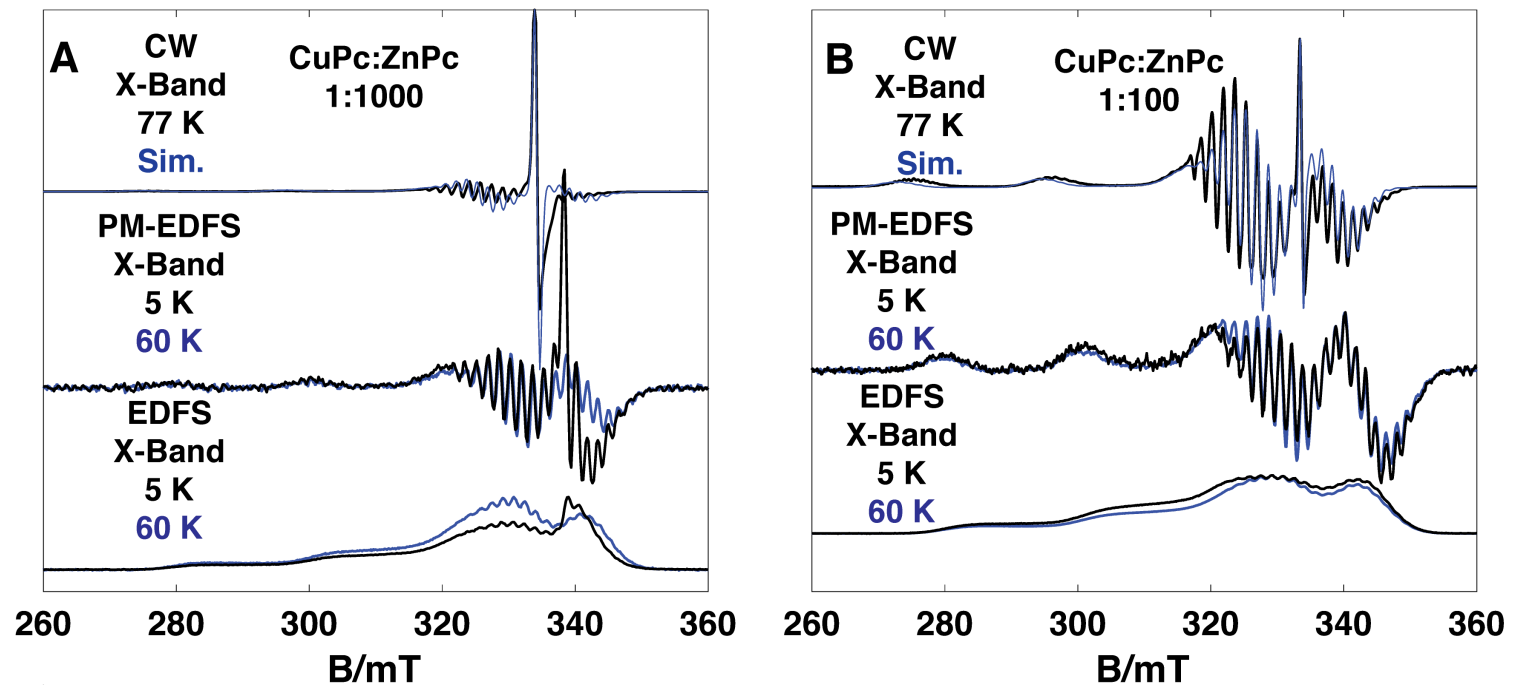

Figure S5. Comparison between CW-EPR at $77 \mathrm{~K}$ to PM-EDFSs and the associated field sweeps at $5 \mathrm{~K}$ (black) and $60 \mathrm{~K}$ (blue) of (A) CuPc:ZnPc 1:1000 and (B) 1:100. In both 1:1000 and 1:100 samples, the radical feature was fit to $\mathrm{a}=2.0$ and a $7.5 \%$ and $0.4 \%$ weight in the CW spectra, respectively, consistent with an $\sim 10 \mathrm{x}$ dilution factor.

\begin{tabular}{|c|c|c|}
\hline & 1:1000 CuPc:ZnPc & 1:100 CuPc:ZnPc \\
\hline $\mathbf{g}_{\|}$ & 2.049 & 2.047 \\
\hline $\mathbf{g}_{\perp}$ & 2.172 & 2.185 \\
\hline$A_{\mathrm{Cu} \|}(\mathrm{MHz})$ & 646.5 & 655.0 \\
\hline$A_{\mathrm{Cu} \|}\left(\mathrm{cm}^{-1}\right)$ & $216 \times 10^{-4}$ & $218 \times 10^{-4}$ \\
\hline$A_{\mathrm{Cu} \perp}(\mathrm{MHz})$ & 15.2 & 35.3 \\
\hline$A_{\mathrm{Cu} \perp}\left(\mathrm{cm}^{-1}\right)$ & $5 \times 10^{-4}$ & $12 \times 10^{-4}$ \\
\hline$A_{N \perp}(M H z)$ & $45-50$ & 45 \\
\hline$A_{N \perp}\left(\mathrm{cm}^{-1}\right)$ & $15-17 \times 10^{-4}$ & $15 \times 10^{-4}$ \\
\hline$A_{N||}(\mathrm{MHz})$ & $40-48$ & 45 \\
\hline$A_{\mathrm{N} \|}\left(\mathrm{cm}^{-1}\right)$ & $13-16 \times 10^{-4}$ & $15 \times 10^{-4}$ \\
\hline
\end{tabular}

Table S2. Best-fit parameters of the simulated EPR spectra in Figure S5 used to reproduce the EPR spectra $\mathrm{T}=77 \mathrm{~K}$. 

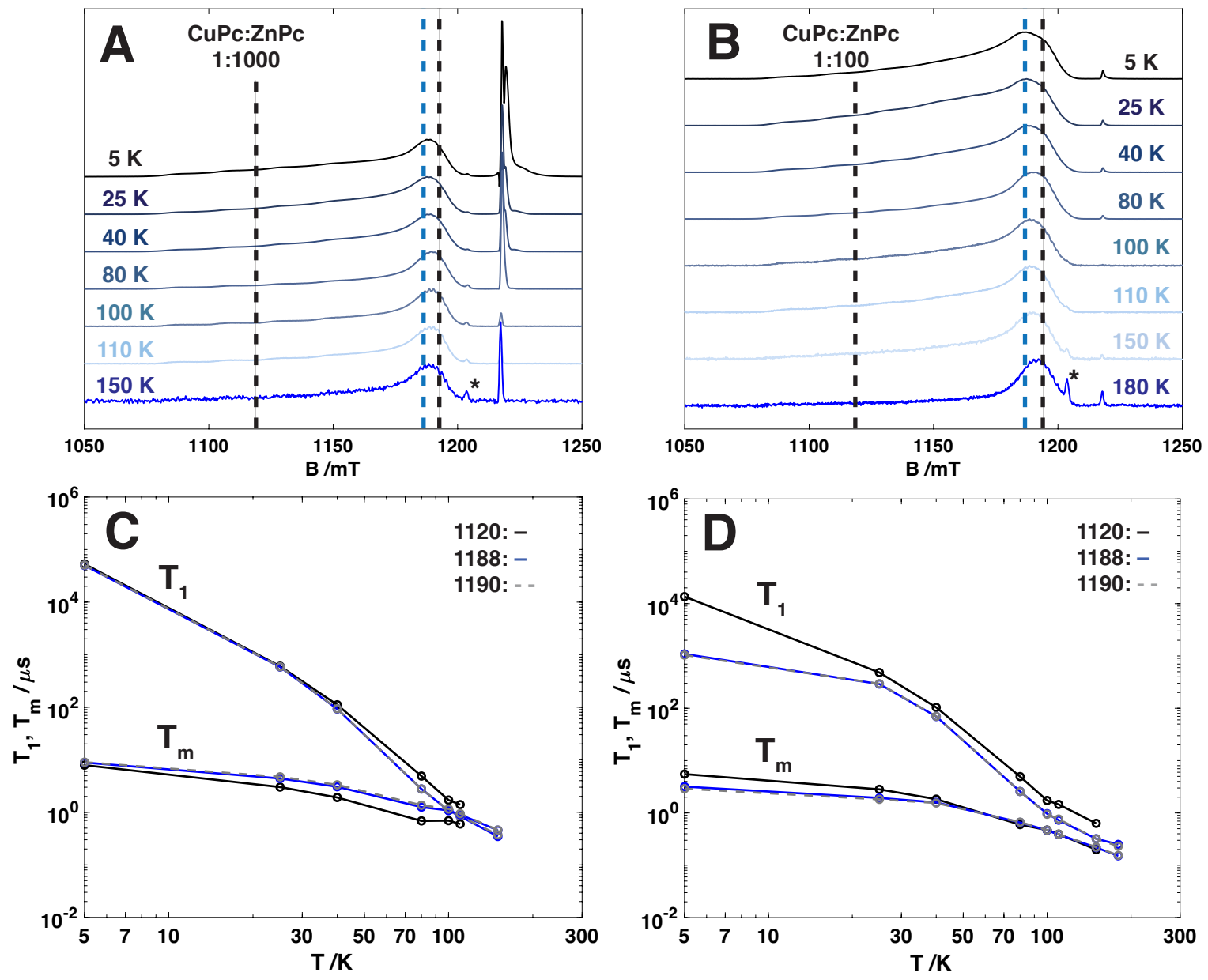

Figure S6. Echo-detected EPR Q-band field sweeps of (A) CuPc 1:1000 and (B) 1:100 from $5 \mathrm{~K}$ to $180 \mathrm{~K}$. Dashed lines indicate field positions where relaxation data were collected. Comparison between the field position dependent behavior of the $T_{1}$ and $T_{m}$ relaxation times from 5 to $180 \mathrm{~K}$ of $\mathrm{CuPc}$ (C) 1:1000 and (D) 1:100. $\left(^{*}\right)$ denotes the sharp radical-like feature at $1204 \mathrm{mT}$ is due to a background signal in the Q-band resonator. 

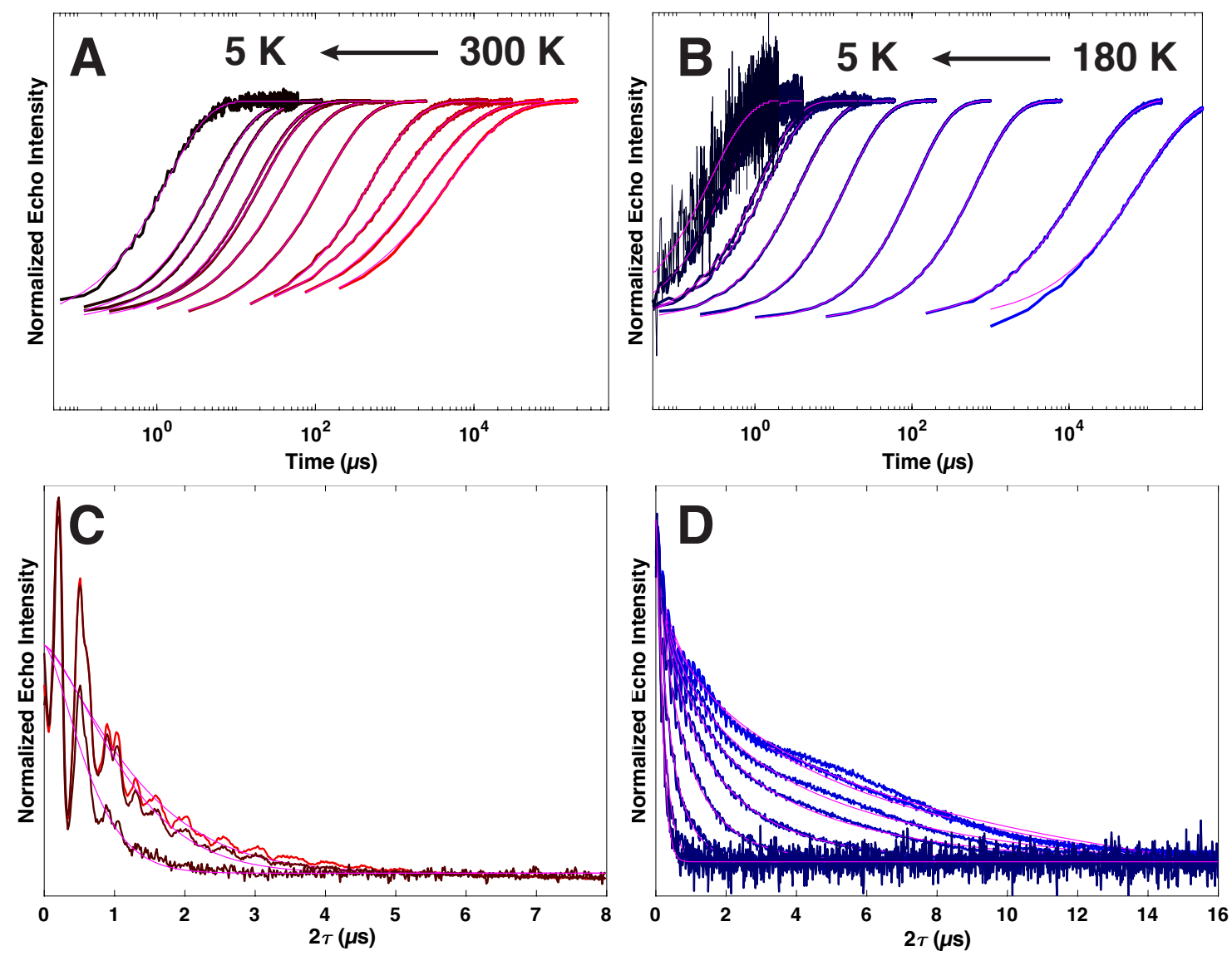

Figure S7. Inversion recoveries and associated fits (pink) of (A) CuPc 1:1000 and (B) VOPc 1:1000 from at X-band. Hahn echo and associated fits (pink) of (C) CuPc 1:1000 and (D) VOPc 1:1000 from at X-band. Due to large ESEEM modulations in VOPc, we have only displayed 3 normalized traces: $5 \mathrm{~K}, 180 \mathrm{~K}$, and 300K. 

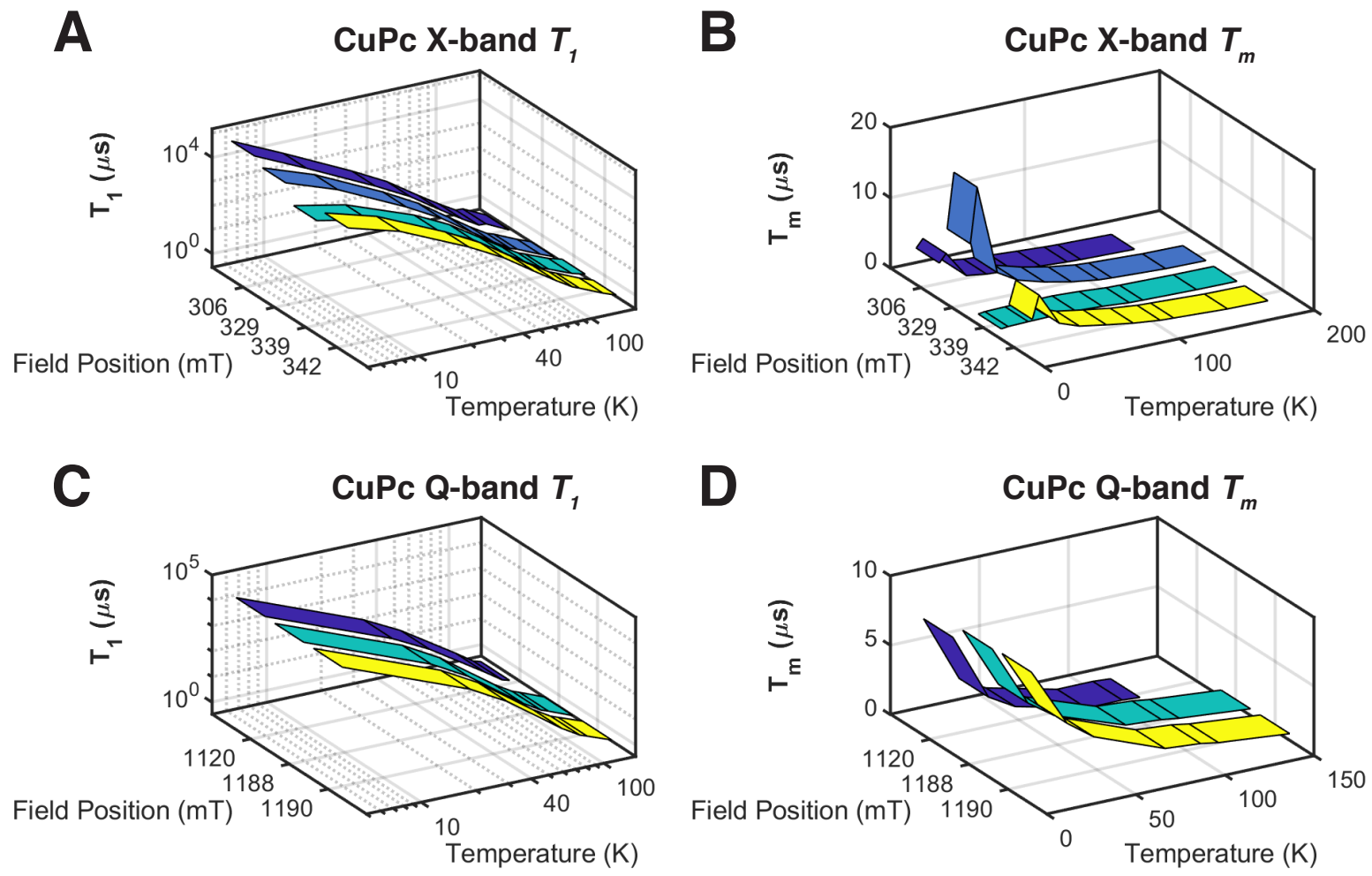

Figure S8. 3D-comparison between the field position dependent behavior of the $\mathrm{CuPc}$ 1:1000 $T_{1}$ and $T_{m}$ relaxation times at X-(A and B) and Q- (C and D) band. 

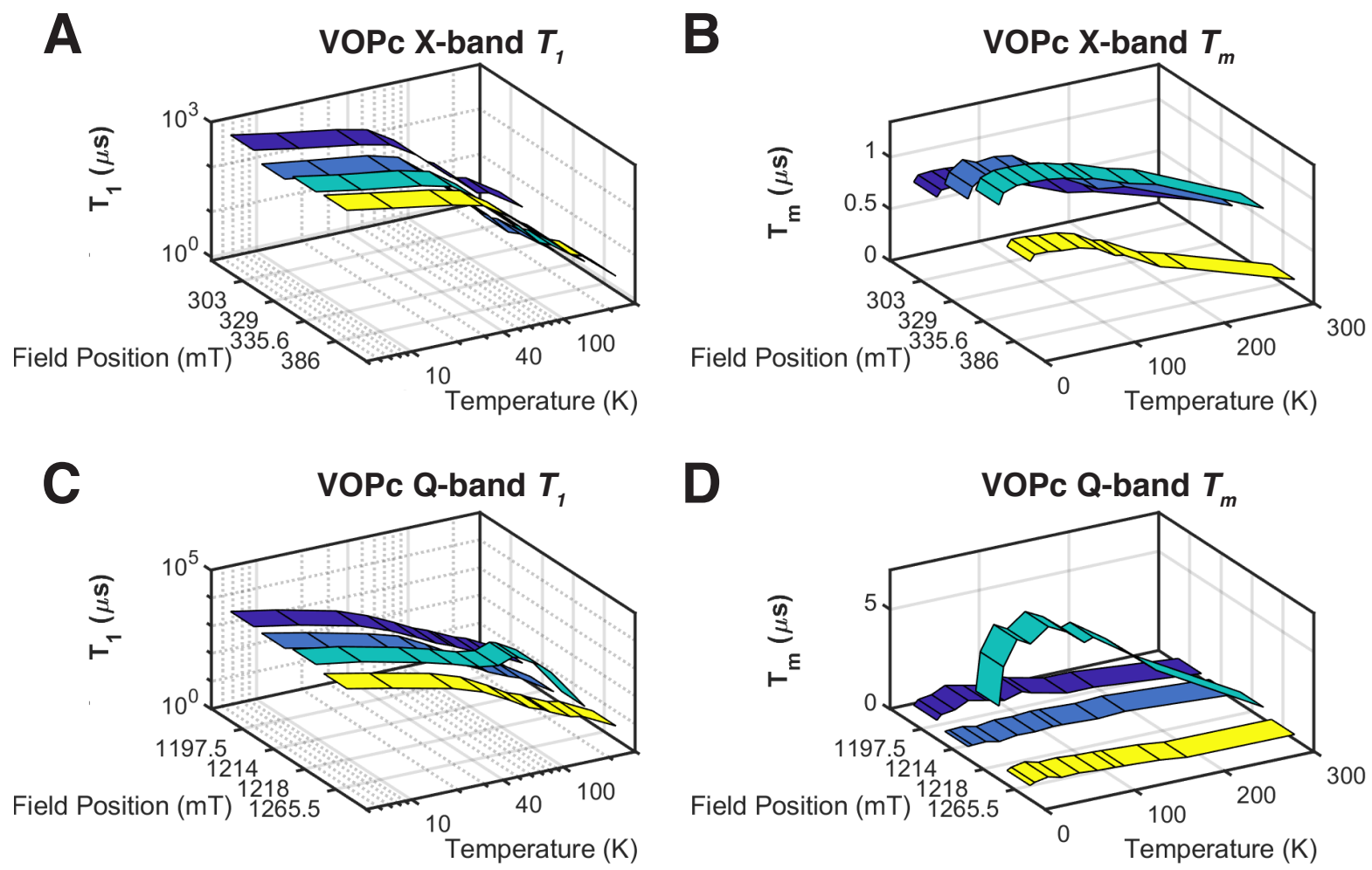

Figure S9. 3D-comparison between the field position dependent behavior of the VOPc 1:1000 $T_{1}$ and $T_{m}$ relaxation times at X- (A and B) and Q- (C and D) band. 
Table S3. 1:1000 VOPc:TiOPc temperature-dependent $T_{1}$ and $T_{m}$ data collected at selected field positions at X-band.

\begin{tabular}{|c|c|c|c|c|c|c|c|c|}
\hline VOPc $1: 1000$ & \multicolumn{2}{|c|}{$303 \mathrm{mT}$} & \multicolumn{2}{|c|}{$329 \mathrm{mT}$} & \multicolumn{2}{|c|}{$335.6 \mathrm{mT}$} & \multicolumn{2}{|c|}{$386 \mathrm{mT}$} \\
\hline Temperature (K) & $\mathrm{T}_{1}(\mu \mathrm{s})$ & $\mathrm{T}_{\mathrm{m}}(\boldsymbol{\mu} \mathrm{s})$ & $\mathrm{T}_{1}(\mu \mathrm{s})$ & $\mathrm{T}_{\mathrm{m}}(\boldsymbol{\mu} \mathrm{s})$ & $\mathbf{T}_{1}(\boldsymbol{\mu} \mathbf{s})$ & $\mathrm{T}_{\mathrm{m}}(\boldsymbol{\mu} \mathrm{s})$ & $\mathrm{T}_{1}(\boldsymbol{\mu} \mathbf{s})$ & $T_{m}(\mu s)$ \\
\hline 5 & $8.2 \cdot 10^{3}$ & 2.7 & $3.3 \cdot 10^{3}$ & 3.4 & $4.9 \cdot 10^{3}$ & 2.9 & $9.6 \cdot 10^{3}$ & 2.6 \\
\hline 10 & $3.6 \cdot 10^{3}$ & 3.1 & $1.8 \cdot 10^{3}$ & 4.0 & $2.4 \cdot 10^{3}$ & 3.4 & $4.2 \cdot 10^{3}$ & 2.9 \\
\hline 25 & $1.4 \cdot 10^{3}$ & 3.2 & $8.7 \cdot 10^{2}$ & 4.3 & $1.1 \cdot 10^{3}$ & 3.6 & $1.5 \cdot 10^{3}$ & 3.2 \\
\hline 40 & $6.4 \cdot 10^{2}$ & 3.2 & $3.4 \cdot 10^{2}$ & 4.5 & $5.0 \cdot 10^{2}$ & 3.7 & $6.2 \cdot 10^{2}$ & 3.1 \\
\hline 60 & $1.9 \cdot 10^{2}$ & 3.1 & $8.8 \cdot 10^{1}$ & 4.4 & $1.3 \cdot 10^{2}$ & 3.6 & $1.7 \cdot 10^{2}$ & 2.9 \\
\hline 80 & $6.5 \cdot 10^{1}$ & 2.9 & $2.9 \cdot 10^{1}$ & 4.0 & $4.2 \cdot 10^{1}$ & 3.4 & $6.1 \cdot 10^{1}$ & 2.7 \\
\hline 100 & $3.5 \cdot 10^{1}$ & 2.6 & $1.4 \cdot 10^{1}$ & 3.7 & $2.1 \cdot 10^{1}$ & 3.3 & $3.3 \cdot 10^{1}$ & 2.4 \\
\hline 110 & $3.1 \cdot 10^{1}$ & 2.5 & $1.2 \cdot 10^{1}$ & 3.6 & $1.8 \cdot 10^{1}$ & 3.2 & $2.7 \cdot 10^{1}$ & 2.3 \\
\hline 150 & $1.7 \cdot 10^{1}$ & 2.1 & 5.5 & 3.1 & 8.5 & 2.8 & $1.3 \cdot 10^{1}$ & 2.0 \\
\hline 180 & $1.1 \cdot 10^{1}$ & 1.8 & 3.4 & 2.7 & 5.4 & 2.6 & 9.6 & 1.6 \\
\hline 300 & 3.4 & - & 0.8 & 1.4 & 1.3 & 1.5 & 2.9 & - \\
\hline
\end{tabular}


Table S4. 1:1000 VOPc:TiOPc temperature-dependent $T_{1}$ and $T_{m}$ data collected at selected field positions at Q-band.

\begin{tabular}{|c|c|c|c|c|c|c|c|c|}
\hline VOPc 1:1000 & \multicolumn{2}{|c|}{$1197.5 m T$} & \multicolumn{2}{|c|}{$1214 m T$} & \multicolumn{2}{c|}{$1218 m T$} & \multicolumn{2}{c|}{$1265.5 m T$} \\
\hline Temperature $(\mathrm{K})$ & $\mathrm{T}_{\mathbf{1}}(\boldsymbol{\mu} \mathbf{s})$ & $\mathrm{T}_{\mathrm{m}}(\boldsymbol{\mu} \mathbf{s})$ & $\mathrm{T}_{1}(\boldsymbol{\mu \mathbf { s }})$ & $\mathrm{T}_{\mathrm{m}}(\boldsymbol{\mu} \mathbf{s})$ & $\mathrm{T}_{1}(\boldsymbol{\mu} \mathbf{s})$ & $\mathrm{T}_{\mathrm{m}}(\boldsymbol{\mu \mathbf { s }})$ & $\mathrm{T}_{1}(\boldsymbol{\mu} \mathbf{s})$ & $\mathrm{T}_{\mathrm{m}}(\boldsymbol{\mu} \mathbf{s})$ \\
\hline 5 & $9.3 \cdot 10^{3}$ & 0.8 & $8.2 \cdot 10^{3}$ & 0.5 & $1.2 \cdot 10^{4}$ & 3.5 & $8.1 \cdot 10^{3}$ & 0.5 \\
\hline 10 & $4.7 \cdot 10^{3}$ & 1.0 & $3.8 \cdot 10^{3}$ & 0.5 & $6.3 \cdot 10^{3}$ & 5.4 & $3.7 \cdot 10^{3}$ & 0.6 \\
\hline 25 & $1.5 \cdot 10^{3}$ & 1.1 & $1.2 \cdot 10^{3}$ & 0.5 & $2.0 \cdot 10^{3}$ & 6.6 & $1.2 \cdot 10^{3}$ & 0.8 \\
\hline 40 & $5.7 \cdot 10^{2}$ & 1.4 & $5.5 \cdot 10^{2}$ & 0.4 & $1.0 \cdot 10^{3}$ & 6.3 & $5.3 \cdot 10^{3}$ & 0.6 \\
\hline 60 & $2.0 \cdot 10^{2}$ & 1.2 & $1.8 \cdot 10^{2}$ & 0.5 & $4.9 \cdot 10^{2}$ & 7.0 & $1.6 \cdot 10^{2}$ & 0.6 \\
\hline 80 & $9.0 \cdot 10^{1}$ & 1.0 & $7.3 \cdot 10^{1}$ & 0.5 & $3.9 \cdot 10^{2}$ & 6.5 & $6.4 \cdot 10^{2}$ & 0.6 \\
\hline 100 & $5.6 \cdot 10^{1}$ & 1.3 & $4.1 \cdot 10^{1}$ & 0.5 & $6.0 \cdot 10^{2}$ & 5.8 & $4.1 \cdot 10^{2}$ & 0.5 \\
\hline 110 & $4.4 \cdot 10^{1}$ & 1.1 & $3.0 \cdot 10^{1}$ & 0.5 & $5.1 \cdot 10^{2}$ & 6.0 & $3.1 \cdot 10^{1}$ & 0.5 \\
\hline 150 & $2.0 \cdot 10^{1}$ & 0.7 & $1.4 \cdot 10^{1}$ & 0.5 & $2.0 \cdot 10^{2}$ & 4.7 & $1.4 \cdot 10^{1}$ & 0.4 \\
\hline 180 & $1.5 \cdot 10^{1}$ & 0.7 & $1.0 \cdot 10^{1}$ & 0.5 & $8.4 \cdot 10^{1}$ & 3.6 & $1.2 \cdot 10^{2}$ & 0.2 \\
\hline 300 & 3.6 & 0.2 & 1.8 & 0.2 & 2.8 & 0.6 & 2.9 & 0.2 \\
\hline
\end{tabular}


Table S5. 1:100 VOPc:TiOPc temperature-dependent $T_{1}$ and $T_{m}$ data collected at selected field positions at X-band.

\begin{tabular}{|c|c|c|c|c|c|c|c|c|}
\hline VOPc $1: 100$ & \multicolumn{2}{|c|}{$303 \mathrm{mT}$} & \multicolumn{2}{|c|}{$329 \mathrm{mT}$} & \multicolumn{2}{|c|}{$335.6 \mathrm{mT}$} & \multicolumn{2}{|c|}{$386 \mathrm{mT}$} \\
\hline Temperature (K) & $\mathrm{T}_{1}(\mu \mathrm{s})$ & $\mathrm{T}_{\mathrm{m}}(\boldsymbol{\mu} \mathbf{s})$ & $\mathrm{T}_{1}(\mu \mathrm{s})$ & $\mathrm{T}_{\mathrm{m}}(\boldsymbol{\mu} \mathrm{s})$ & $\mathbf{T}_{1}(\boldsymbol{\mu} \mathbf{s})$ & $\mathrm{T}_{\mathrm{m}}(\boldsymbol{\mu} \mathrm{s})$ & $\mathrm{T}_{1}(\boldsymbol{\mu} \mathbf{s})$ & $T_{m}(\mu s)$ \\
\hline 5 & $1.0 \cdot 10^{3}$ & 0.9 & $6.3 \cdot 10^{2}$ & 1.0 & $9.3 \cdot 10^{2}$ & 1.2 & $1.0 \cdot 10^{3}$ & 0.8 \\
\hline 10 & $6.6 \cdot 10^{2}$ & 0.9 & $4.6 \cdot 10^{2}$ & 1.2 & $6.2 \cdot 10^{2}$ & 1.3 & $6.8 \cdot 10^{2}$ & 0.9 \\
\hline 25 & $4.1 \cdot 10^{2}$ & 0.9 & $3.0 \cdot 10^{2}$ & 1.2 & $3.9 \cdot 10^{2}$ & 1.3 & $4.1 \cdot 10^{2}$ & 0.9 \\
\hline 40 & $2.9 \cdot 10^{2}$ & 0.9 & $2.0 \cdot 10^{2}$ & 1.2 & $2.7 \cdot 10^{2}$ & 1.3 & $2.8 \cdot 10^{2}$ & 0.9 \\
\hline 60 & $1.3 \cdot 10^{2}$ & 0.9 & $6.9 \cdot 10^{1}$ & 1.2 & $1.0 \cdot 10^{2}$ & 1.3 & $1.2 \cdot 10^{2}$ & 0.9 \\
\hline 80 & $5.4 \cdot 10^{1}$ & 0.9 & $2.7 \cdot 10^{1}$ & 1.2 & $4.2 \cdot 10^{1}$ & 1.3 & $5.2 \cdot 10^{1}$ & 0.8 \\
\hline 100 & $2.8 \cdot 10^{1}$ & 0.8 & $1.2 \cdot 10^{1}$ & 1.1 & $2.0 \cdot 10^{1}$ & 1.3 & $2.8 \cdot 10^{1}$ & 0.7 \\
\hline 110 & $2.5 \cdot 10^{1}$ & 0.8 & $1.0 \cdot 10^{1}$ & 1.1 & $1.7 \cdot 10^{1}$ & 1.2 & $2.3 \cdot 10^{1}$ & 0.7 \\
\hline 150 & 9.3 & 0.6 & 3.5 & 0.9 & 5.7 & 1.1 & 8.9 & 0.5 \\
\hline 180 & 8.1 & 0.6 & 2.8 & 0.8 & 4.4 & 1.1 & 7.0 & 0.4 \\
\hline 300 & 2.7 & 0.3 & 0.8 & 0.4 & 1.3 & 0.6 & 1.7 & 0.1 \\
\hline
\end{tabular}


Table S6. 1:1000 CuPc:ZnPc temperature-dependent $T_{1}$ and $T_{m}$ data collected at selected field positions at X-band.

\begin{tabular}{|c|c|c|c|c|c|c|c|c|}
\hline CuPc1:1000 & \multicolumn{2}{|c|}{$306 m T$} & \multicolumn{2}{|c|}{$329 m T$} & \multicolumn{2}{c|}{$339 m T$} & \multicolumn{2}{c|}{$342 m T$} \\
\hline Temperature $(\mathrm{K})$ & $\mathrm{T}_{1}(\boldsymbol{\mu \mathbf { s }})$ & $\mathrm{T}_{\mathrm{m}}(\boldsymbol{\mu \mathbf { s }})$ & $\mathrm{T}_{1}(\boldsymbol{\mu \mathbf { s }})$ & $\mathrm{T}_{\mathrm{m}}(\boldsymbol{\mu} \mathbf{s})$ & $\mathrm{T}_{1}(\boldsymbol{\mu s})$ & $\mathrm{T}_{\mathrm{m}}(\boldsymbol{\mu s})$ & $\mathrm{T}_{1}(\boldsymbol{\mu s})$ & $\mathrm{T}_{\mathrm{m}}(\boldsymbol{\mu s})$ \\
\hline 5 & $1.6 \cdot 10^{5}$ & 4.4 & $8.3 \cdot 10^{4}$ & $1.0 \cdot 10^{1}$ & $1.5 \cdot 10^{4}$ & 0.7 & $5.1 \cdot 10^{4}$ & 5.0 \\
\hline 10 & $1.7 \cdot 10^{4}$ & 5.5 & $1.5 \cdot 10^{4}$ & $1.8 \cdot 10^{1}$ & $5.6 \cdot 10^{3}$ & 0.8 & $1.3 \cdot 10^{4}$ & 8.1 \\
\hline 25 & $7.4 \cdot 10^{2}$ & 1.8 & $6.7 \cdot 10^{2}$ & 5.0 & $5.0 \cdot 10^{2}$ & 0.9 & $6.8 \cdot 10^{2}$ & 3.5 \\
\hline 40 & $1.3 \cdot 10^{2}$ & 1.4 & $1,1 \cdot 10^{2}$ & 3.4 & $9.7 \cdot 10^{1}$ & 1.0 & $1.2 \cdot 10^{2}$ & 2.5 \\
\hline 60 & $1.9 \cdot 10^{1}$ & 1.1 & $1.5 \cdot 10^{1}$ & 2.3 & $1.5 \cdot 10^{1}$ & 1.0 & $1.7 \cdot 10^{1}$ & 1.9 \\
\hline 80 & 5.1 & 0.9 & 3.9 & 1.5 & 4.0 & 0.8 & 4.5 & 1.3 \\
\hline 100 & 2.0 & 0.7 & 1.5 & 0.9 & 1.5 & 0.5 & 1.7 & 0.9 \\
\hline 110 & 1.9 & 0.6 & 1.2 & 0.8 & 1.2 & 0.5 & 1.3 & 0.8 \\
\hline 150 & 0.7 & 0.4 & 0.4 & 0.5 & 0.5 & 0.3 & 0.5 & 0.4 \\
\hline 180 & - & - & 0.3 & 0.4 & 0.3 & 0.3 & 0.3 & 0.4 \\
\hline
\end{tabular}


Table S7. 1:1000 CuPc:ZnPc temperature-dependent $T_{1}$ and $T_{m}$ data collected at selected field positions at Q-band.

\begin{tabular}{|c|c|c|c|c|c|c|}
\hline CuPc1:1000 & \multicolumn{2}{|c|}{$1120 m T$} & \multicolumn{2}{c|}{$1188 m T$} & \multicolumn{2}{c|}{$1190 m T$} \\
\hline Temperature $(\mathrm{K})$ & $\mathrm{T}_{1}(\boldsymbol{\mu} \mathbf{s})$ & $\mathrm{T}_{\mathrm{m}}(\boldsymbol{\mu} \mathbf{s})$ & $\mathrm{T}_{1}(\boldsymbol{\mu \mathbf { s }})$ & $\mathrm{T}_{\mathrm{m}}(\boldsymbol{\mu \mathbf { s }})$ & $\mathrm{T}_{1}(\boldsymbol{\mu} \mathbf{s})$ & $\mathrm{T}_{\mathrm{m}}(\boldsymbol{\mu} \mathbf{s})$ \\
\hline 5 & $5.3 \cdot 10^{4}$ & 7.9 & $5.0 \cdot 10^{4}$ & 8.9 & $5.1 \cdot 10^{4}$ & 9.0 \\
\hline 25 & $6.0 \cdot 10^{2}$ & 3.0 & $5.8 \cdot 10^{2}$ & 4.4 & $5.9 \cdot 10^{2}$ & 4.7 \\
\hline 40 & $1.1 \cdot 10^{2}$ & 1.9 & $9.2 \cdot 10^{1}$ & 3.1 & $9.3 \cdot 10^{1}$ & 3.3 \\
\hline 80 & 4.9 & 0.7 & 2.8 & 1.3 & 2.8 & 1.4 \\
\hline 100 & 1.7 & 0.7 & 1.1 & 1.1 & 1.1 & 1.2 \\
\hline 110 & 1.4 & 0.6 & 0.8 & 0.9 & 0.8 & 0.9 \\
\hline 150 & - & - & 0.3 & 0.5 & 0.4 & 0.5 \\
\hline
\end{tabular}


Table S8. 1:100 CuPc:ZnPc temperature-dependent $T_{1}$ and $T_{m}$ data collected at selected field positions at X-band.

\begin{tabular}{|c|c|c|c|c|c|c|c|c|}
\hline CuPc1:100 & \multicolumn{2}{|c|}{$306 m T$} & \multicolumn{2}{|c|}{$329 m T$} & \multicolumn{2}{c|}{$339 m T$} & \multicolumn{2}{c|}{$342 m T$} \\
\hline Temperature $(\mathrm{K})$ & $\mathrm{T}_{1}(\boldsymbol{\mu s})$ & $\mathrm{T}_{\mathrm{m}}(\boldsymbol{\mu s})$ & $\mathrm{T}_{1}(\boldsymbol{\mu} \mathbf{s})$ & $\mathrm{T}_{\mathrm{m}}(\boldsymbol{\mu} \mathbf{s})$ & $\mathrm{T}_{1}(\boldsymbol{\mu s})$ & $\mathrm{T}_{\mathrm{m}}(\boldsymbol{\mu s})$ & $\mathrm{T}_{1}(\boldsymbol{\mu s})$ & $\mathrm{T}_{\mathrm{m}}(\boldsymbol{\mu s})$ \\
\hline 5 & $1.2 \cdot 10^{4}$ & 1.4 & $2.7 \cdot 10^{3}$ & 1.1 & $3.7 \cdot 10^{3}$ & 1.5 & $5.6 \cdot 10^{3}$ & 1.5 \\
\hline 10 & $5.5 \cdot 10^{3}$ & 1.4 & $1.9 \cdot 10^{3}$ & 1.2 & $2.4 \cdot 10^{3}$ & 1.5 & $3.7 \cdot 10^{3}$ & 1.5 \\
\hline 25 & $6.0 \cdot 10^{2}$ & 1.3 & $4.0 \cdot 10^{2}$ & 1.2 & $4.7 \cdot 10^{2}$ & 1.5 & $5.6 \cdot 10^{2}$ & 1.5 \\
\hline 40 & $1.2 \cdot 10^{2}$ & 1.2 & $8.9 \cdot 10^{1}$ & 1.1 & $9.5 \cdot 10^{1}$ & 1.4 & $1.1 \cdot 10^{2}$ & 1.3 \\
\hline 60 & $2.0 \cdot 10^{1}$ & 0.9 & $1.4 \cdot 10^{1}$ & 0.9 & $1.4 \cdot 10^{1}$ & 1.1 & $1.5 \cdot 10^{1}$ & 1.0 \\
\hline 80 & 5.3 & 0.7 & 3.9 & 0.7 & 3.7 & 0.7 & 4.1 & 0.7 \\
\hline 100 & 2.5 & 0.5 & 1.7 & 0.5 & 1.6 & 0.5 & 1.8 & 0.5 \\
\hline 110 & 1.7 & 0.4 & 1.2 & 0.4 & 1.2 & 0.5 & 1.3 & 0.5 \\
\hline 150 & 0.8 & 0.3 & 0.5 & 0.3 & 0.5 & 0.3 & 0.5 & 0.3 \\
\hline 180 & - & - & 0.3 & 0.2 & 0.3 & 0.2 & 0.3 & 0.2 \\
\hline
\end{tabular}


Table S9. 1:100 CuPc:ZnPc temperature-dependent $T_{1}$ and $T_{m}$ data collected at selected field positions at Q-band.

\begin{tabular}{|c|c|c|c|c|c|c|}
\hline CuPc1:100 & \multicolumn{2}{|c|}{$1120 m T$} & \multicolumn{2}{c|}{$1188 m T$} & \multicolumn{2}{c|}{$1190 m T$} \\
\hline Temperature $(\mathrm{K})$ & $\mathrm{T}_{1}(\boldsymbol{\mu s})$ & $\mathrm{T}_{\mathrm{m}}(\boldsymbol{\mu s})$ & $\mathrm{T}_{1}(\boldsymbol{\mu s})$ & $\mathrm{T}_{\mathrm{m}}(\boldsymbol{\mu s})$ & $\mathrm{T}_{\mathbf{1}}(\boldsymbol{\mu} \mathbf{s})$ & $\mathrm{T}_{\mathrm{m}}(\boldsymbol{\mu s})$ \\
\hline 5 & $1.36 \cdot 10^{4}$ & 5.5 & $1.1 \cdot 10^{3}$ & 3.2 & $1.0 \cdot 10^{3}$ & 2.9 \\
\hline 25 & $4.8 \cdot 10^{2}$ & 2.8 & $2.9 \cdot 10^{2}$ & 1.9 & $3.0 \cdot 10^{2}$ & 1.8 \\
\hline 40 & $1.0 \cdot 10^{2}$ & 1.8 & $7.0 \cdot 10^{1}$ & 1.6 & $7.0 \cdot 10^{1}$ & 1.5 \\
\hline 80 & 4.9 & 0.6 & 2.6 & 0.7 & 2.6 & 0.7 \\
\hline 100 & 1.7 & 0.5 & 1.0 & 0.5 & 0.9 & 0.5 \\
\hline 110 & 1.5 & 0.4 & 0.7 & 0.4 & 0.8 & 0.4 \\
\hline 150 & 0.6 & 0.2 & 0.3 & 0.2 & 0.3 & 0.2 \\
\hline 180 & - & - & 0.3 & 0.2 & 0.2 & 0.2 \\
\hline
\end{tabular}



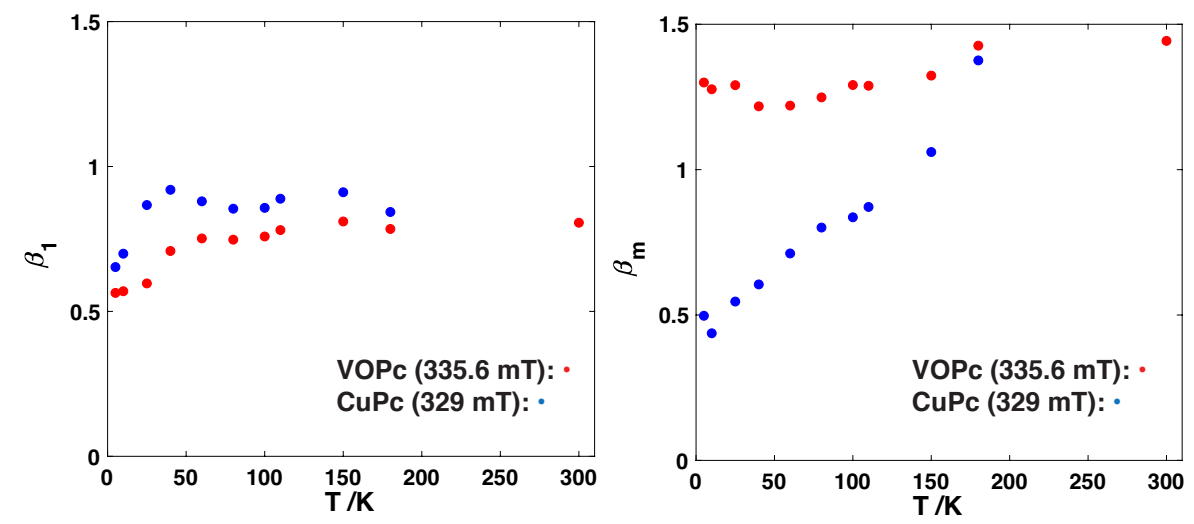

Figure S10. Comparison stretching term, $\beta$, for VOPc 1:1000 at $335.6 \mathrm{mT}$ (red) and CuPc 1:1000 at $329 \mathrm{mT}$ (blue) (A) $T_{1}$ times $\left(\beta_{1}\right)$ and (B) $T_{m}$ times $\left(\beta_{\mathrm{m}}\right)$. 

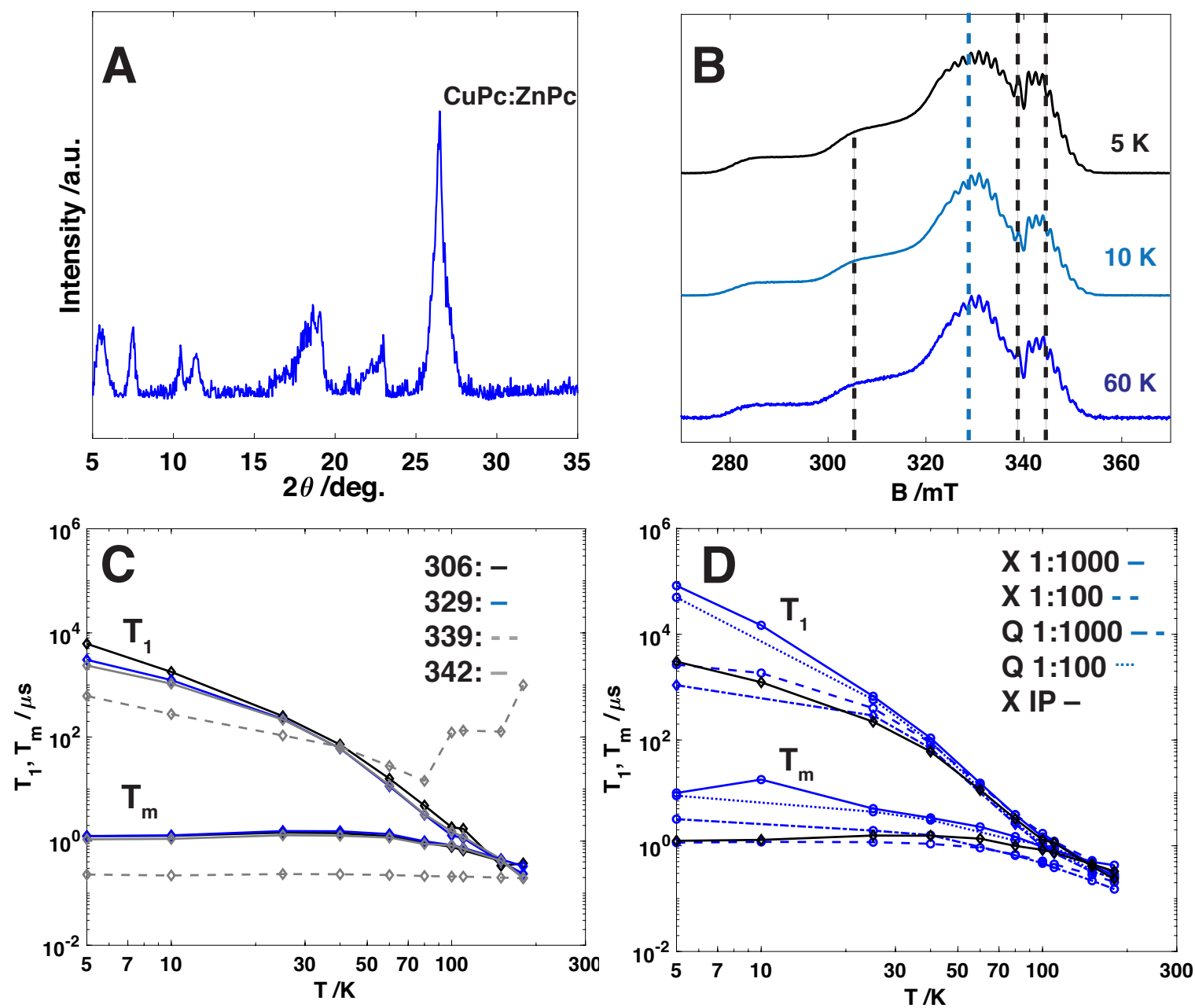

Figure S11. (A) Comparison of the PXRD pattern of an independent preparation of CuPc:ZnPc. (B) Echo-detected EPR X-band field sweeps of CuPc at $5 \mathrm{~K}, 10 \mathrm{~K}$, and $60 \mathrm{~K}$. Dashed lines indicate field positions where relaxation data were collected. (C) Comparison of the field position dependent behavior of the $T_{1}$ and $T_{m}$ relaxation times from 5-180 K of CuPc dilution. (D) Comparison between X-band $T_{1}$ and $T_{m}$ times vs temperature for the $329 \mathrm{mT}$ features in CuPc at X-and Q-band for the 1:1000, 1:100 preparations and the independent preparation.

A CuPc:ZnPc sample was prepared to yield a final dilution of 1:1000 by an independent method analogous to the preparation for VOPc:TiOPc samples using DCM/CF $3 \mathrm{CO}_{2} \mathrm{H} / \mathrm{IPA}$. Due to solubility issues in the preparation of this CuPc:ZnPc sample, the final concentration is 
unknown. The PXRD patterns are distinct from the 1:1000 and 1:100 previously prepared (Figure S11A). However, the CW-EPR spectrum shows similar copper features despite a significantly more intense radical signal (Figure S12). As well, the $T_{1}$ and $T_{m}$ temperaturedependences observed for this sample were consistent with the other CuPc samples when measured at 306, 329, and $342 \mathrm{mT}$ (Figure S11). This preparation method did, however, display substantially different behavior with respect to the $339 \mathrm{mT}$ field position. In the EDFS, there is a sharp signal at $339 \mathrm{mT}$. The $T_{1}$ times measured at this position remained significantly longer at higher temperatures than for other field positions, whereas the $T_{m}$ times of this feature were found to be lower than at the other positions. These observations indicate that this signal arises from the organic radical. In contrast to the samples prepared by the sulfuric acid method, the EDFS spectra do not change much with increasing temperature. The distinct temperature dependences in the EDFS spectra, $T_{1}$, and $T_{m}$ times between the various preparations indicate that the radical species may be delocalized in different ways in these two samples. When monitoring relaxation behavior at field positions not associated with the radical (e.g. 306, $329 \mathrm{mT}$ at X-band), then similar temperaturedependent trends are observed regardless of sample preparation method or concentration (Figure S11D). This suggests that the radical is not the dominant feature contributing to the differences in relaxation times between VOPc and CuPc mixtures. 


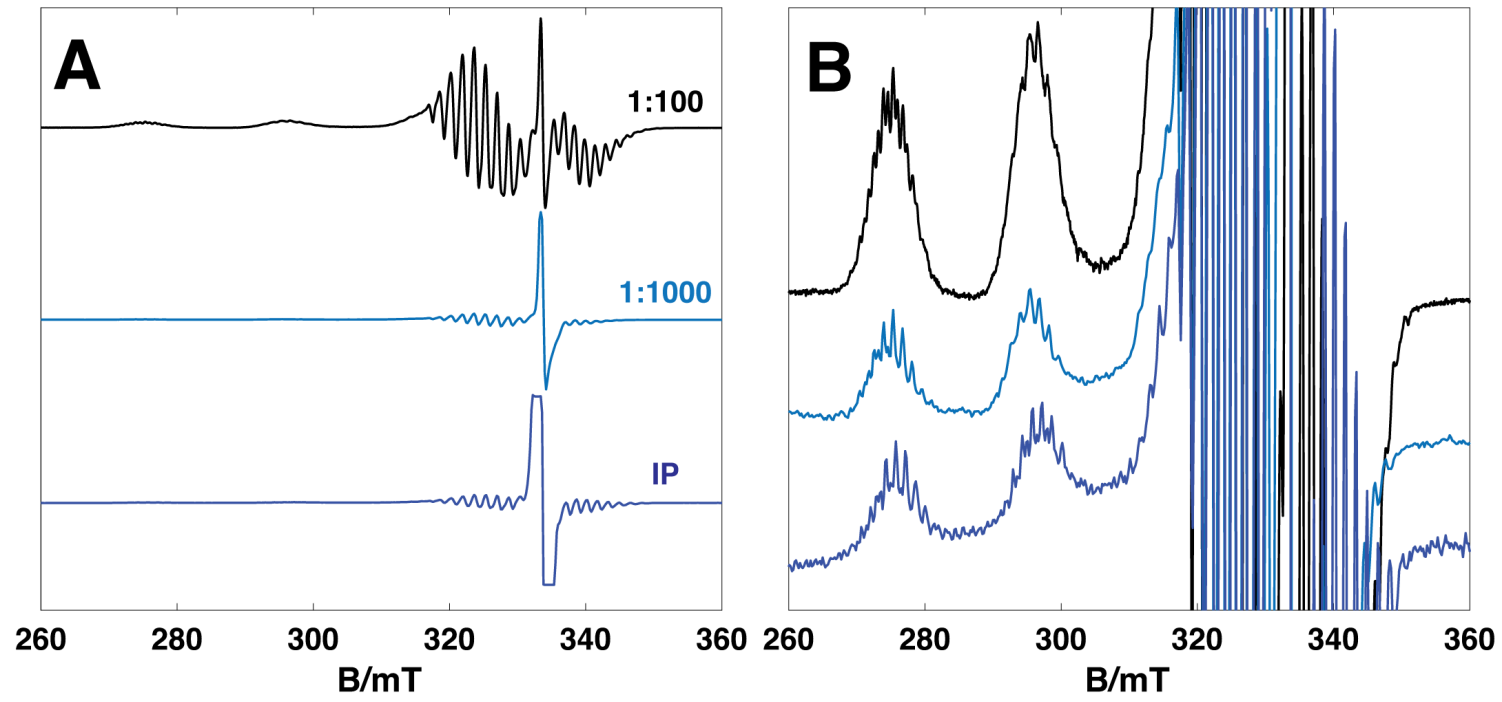

Figure S12. (A) Comparison of CW-EPR at $77 \mathrm{~K}$ of the CuPc:ZnPc 1:100 and 1:1000 and independent preparations. (B) Zoom-in to show ${ }^{14} \mathrm{~N}$ superhyperfine features. 


\section{References:}

(1) Schmidt, M. U. Industrial Organic Pigments; 2019; pp 834-842. https://doi.org/10.1107/97809553602060000984.

(2) Atzori, M.; Tesi, L.; Morra, E.; Chiesa, M.; Sorace, L.; Sessoli, R. Room-Temperature Quantum Coherence and Rabi Oscillations in Vanadyl Phthalocyanine: Toward Multifunctional Molecular Spin Qubits. J. Am. Chem. Soc. 2016, 138 (7), 2154-2157. https://doi.org/10.1021/jacs.5b13408.

(3) Assour, J. M.; Harrison, S. E. On the Origin of Unpaired Electrons in Metal-Free Phthalocyanine. J. Phys. Chem. 1964, 68 (4), 872-876.

https://doi.org/10.1021/j100786a029. 